\title{
Introduction to the vol. 46, no. 2, 2019
}

\section{Maomi Ueno ${ }^{1}$}

Published online: 24 September 2019

(c) The Behaviormetric Society 2019

Welcome to the vol. 46, no. 2, 2019 of Behaviormetrika. In this issue, we have the following eight original papers, one short note and one invited paper.

This issue includes a special feature: "Dimension reduction and cluster analysis" (Vichi et al. 2019; Durieux and Wilderjans 2019; Yamagish et al. 2019; D’Ambrosio and Heiser 2019; van der Hoef and Warrens 2019) which was edited by Michel van de Velden, Alfonso Iodice D'Enza and Michio Yamamoto.

This special feature specifically addresses state-of-the-art clustering methods.

The regular papers of this issue include the following four original papers and one short note.

- The original paper "Bayesian model checking in cognitive diagnostic models" by Nan Wang and Russell Alomond (2019) proposes to use prior predictive posterior simulation method and posterior predictive method to investigate the person fit of DINA model (the deterministic inputs, noisy "and" gate) (Haertel 1989; Junker and Sijtsma 2001). The rationale of the Bayesian model checking method is to compare the discrepancy measure that calculates with the observed data to a distribution obtained by applying it to multiple simulated data sets. The result of this study might help researchers to choose the appropriate statistic indices to identify different types of person misfit including guessing, random guessing, sleeping, and cheating.

- The short note "Decline of Pearson's $r$ with categorization of variables: a largescale simulation', by Onoshima et al. (2019) examines the degree of bias of correlation coefficients computed from categorical variables. The results revealed more serious biases than highlighted in previous studies.

- The original paper "Consequences of ignoring nested data structure on item parameters in Rasch/1P-IRT mode' by Miyazaki et al. (2019) examines the consequences of ignoring a nested data structure on the Rasch/one parameter item response theory model. Simulation experiments demonstrate negative consequences in estimating the standard errors, although the point estimates were unbiased and identical to the ones from the three level analysis. A real

Maomi Ueno

ueno@ai.is.uec.ac.jp

1 University of Electro-Communications, Tokyo, Japan 
dataset from the IEA civic education study in 1999 was used to illustrate the simulation results.

- The original paper "Bayesian modeling for overdispersed event-count time series" by Fukumoto et al. (2019) addresses one of the state-of-the-art methods for event-count time-series data analyses, the Poisson exponentially weighted moving average (P-EWMA) model. It has been known to lead to incorrect inference in the presence of omitted variables even if they are not confounding. To tackle this problem, this paper proposes a negative binomial integrated error [NB-I(1)] model, which can be estimated via Markov Chain Monte Carlo methods. Simulations show that when the data are generated by a P-EWMA model, but an non-confounding covariate is omitted at the stage of estimation, the P-EWMA model's credible interval is optimistically too narrow to contain the true value at the nominal level, whereas the NB-I(1) model does not suffer this problem. To explore the models' performance, this paper replicates a study on an annual count of militarized interstate disputes.

- The original paper "The Application of Minimum Discrepancy Estimation in Implementation of Cognitive Diagnostic Models" by Dai et al. (2019) studies cognitive diagnostic models (CDMs) in operational assessments. This study introduces two parameter estimation methods, the minimum discrepancy (MD) and the minimum discrepancy maximum likelihood (MDML) which were originally proposed in the knowledge space theory (KST), to the context of CDMs. Numerical results demonstrate the effectiveness of applying MD and MDML in CDM parameter estimations, especially for the small sample sizes.

\section{References}

D’Ambrosio A, Heiser WJ (2019) A distribution-free soft-clustering method for preference rankings. Behaviormetrika 42(2):1-19

Dai S, Wang X, Svetina D (2019) The application of minimum discrepancy estimation in implementation of cognitive diagnostic models. Behaviormetrika 42(2):1-29

Durieux J, Wilderjans TF (2019) Partitioning subjects based on high-dimensional fMRI data: comparison of several clustering methods and studying the influence of ICA data reduction in big data. Behaviormetrika 42(2):1-41

Fukumoto K, Beger A, Moore WH (2019) Bayesian modeling for overdispersed event-count time series. Behaviormetrika 42(2):1-18

Haertel EH (1989) Using restricted latent class models to map the skill structure of achievement items. J Educ Meas 26(4):333-352

Junker BW, Sijtsma K (2001) Cognitive assessment models with few assumptions and connections with nonparametric item response theory. Appl Psychol Meas 25(3):258-272

Miyazaki Y, Chungbaek Y, Shropshire KO, Hedeker D (2019) Consequences of ignoring nested data structure on item parameters in Rasch/1P-IRT model. Behaviormetrika 42(2):1-34

Onoshima T, Shiina K, Ueda T, Kubo S (2019) Decline of Pearson's $r$ with categorization of variables: a large-scale simulation. Behaviormetrika 42(2):1-11 
van der Hoef H, Warrens MJ (2019) Understanding information theoretic measures for comparing clusterings. Behaviormetrika 42(2):1-18

Vichi M, Vicari D, Kiers HAL (2019) Clustering and dimension reduction for mixed variables. Behaviormetrika 42(2):1-27

Yamagish Y, Tabnioka K, Yadohisa H (2019) Constrained nonmetric principal component analysis. Behaviormetrika 42(2):1-20

Publisher's Note Springer Nature remains neutral with regard to jurisdictional claims in published maps and institutional affiliations. 